\title{
Retraction Note: Analysis of the dynamics of laser induced plume propagation from liquid matrix using fast photography
}

\author{
Xing Fan $^{1} \cdot$ Jian Xiao ${ }^{1} \cdot$ Guang-Zhou Hu ${ }^{1} \cdot$ Yun-Peng Zhao ${ }^{1}$. Shi-Gang Kang ${ }^{1,2}$ - Jin-Li Lu ${ }^{1} \cdot$ Hong-Ru Fan ${ }^{1}$
}

Published online: 15 October 2019

(C) Institute of Chemistry, Slovak Academy of Sciences 2019

\section{Retraction Note: Chemical Papers 70 (6) 848-855 (2016) https://doi.org/10.1515/chempap-2016-0012}

The editor has retracted this article [1] because it has significant overlap with a work published by Fan and Murray [2] and is therefore redundant. Xing Fan does not agree to this retraction. Jian Xiao, Guang-Zhou Hu, Yun-Peng Zhao, ShiGang Kang, Jin-Li Lu and Hong-Ru Fan have not responded to any correspondence from the editor about this retraction.

[1] Fan, X., Xiao, J., Hu, GZ. et al. Chem. Pap. (2016) 70: 848. https://doi.org/10.1515/chempap-2016-0012

[2] Fan, X and K Murray. J. Phys. Chem. A. (2010) 3: 114. https://doi.org/10.1021/jp9077163

The original article can be found online at https://doi.org/10.1515/ chempap-2016-0012.

Xing Fan

fanxing@cumt.edu.cn

$\triangle$ Shi-Gang Kang

kangshigang@ahut.edu.cn

1 Key Laboratory of Coal Processing and Efficient Utilization (Ministry of Education), China University of Mining \& Technology, Xuzhou 221116, Jiangsu, China

2 School of Chemistry and Chemical Engineering, Anhui University of Technology, Ma' anshan 243002, Anhui, China 